\title{
Angiotensin-converting enzyme inhibition therapy in heart failure: Practical guidelines
}

\author{
ROBERT DIBIANCO, M.D
} Takoma Park, Maryland

\begin{abstract}
At present, captopril is the only vasodilator indicated for the treatment of congestive heart failure. It is increasingly being used to treat those patients who are nonresponsive to conventional therapy with digitalis and diuretics. Patients most likely to benefit from captopril therapy are those with left heart failure caused by myocardial damage (i.e., dilated cardiomyopathies) and left ventricular overload disorders. The drug allows the practitioner to directly block the renin-angiotensinaldosterone system and to counteract vasoconstriction and fluid retention mechanisms, which are critical factors in the syndrome of heart failure. Working through several mechanisms, the most important of which is to inhibit the angiotensin-converting enzyme (ACE), captopril has been proved to increase exercise capacity and to alleviate the symptoms (dyspnea and orthopnea, fatigue, edema) associated with CHF. In this use, captopril has produced reductions in arrhythmia, decreases in diuretic requirements, and improvements in renal function. The role of this ACE inhibitor in prolonging the lives of CHF patients has not yet been established, although preliminary suggestions of a beneficial effect from uncontrolled evaluations appear promising.
\end{abstract}

When the heart cannot meet the metabolic and circulatory needs of the body, congestive heart failure $(\mathrm{CHF})$ is present. No matter what the cause-myocardial disease, damage, or excessive workload-the compensatory mechanisms that are triggered to maintain cardiac output and blood pressure eventually become detrimental (Fig. 1).

Increased sympathetic nervous system activity in CHF stimulates both beta- and alpha-adrenergic receptors. The beta-adrenoreceptor-mediated responses cause a faster heart rate and stronger force of myocardial contraction, whereas the alpha-adrenoreceptor responses include arterial vasoconstriction (causing increased afterload) and venoconstriction (resulting in increased preload).

CHF also activates the renin-angiotensin-aldosterone (RAA) system (Fig. 2). There is an initial release of the enzyme renin, which triggers the transformation of angiotensinogen into the inactive precursor, angiotensin I. The angiotensin-converting enzyme (ACE) then converts angiotensin I into an extremely potent vasoconstrictor, angioten$\sin$ II. Afterload is increased via the direct systemic vasoconstriction caused by angiotensin II, while preload is increased primarily through the stimulation of aldosterone secretion, which causes sodium and water retention, and, therefore, expanded blood volume.

Increases in preload and cardiac contractility from both of these compensatory mechanisms increase cardiac output. Vasoconstriction, however, may cause a reduction in cardiac output, and fluid retention exacerbates respiratory work. Further reductions in cardiac output produce additional secretions of vasoconstrictor hormones, resulting in a vicious cycle (Fig. 1).

\section{Present therapy in CHF}

Standard treatment of CHF consists of digitalis, to improve myocardial contractility, and diuretics, to eliminate sodium and fluid retention. For many patients, however, these medications will not provide relief from inexorably worsening symptoms, and further treatment is required to allow the patient to function more effectively on a daily basis. In these patients, the balanced vasodilation available with captopril can produce beneficial results when added to standard therapy (Table 1). 


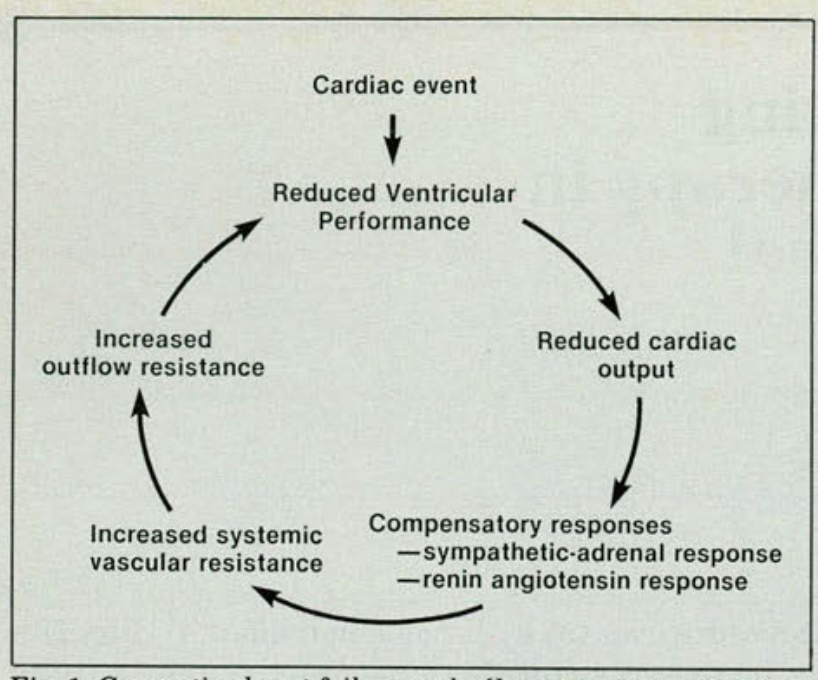

Fig. 1. Congestive heart failure cycle. Compensatory systems are triggered to maintain cardiac output in the failing heart, with eventual detrimental effects.

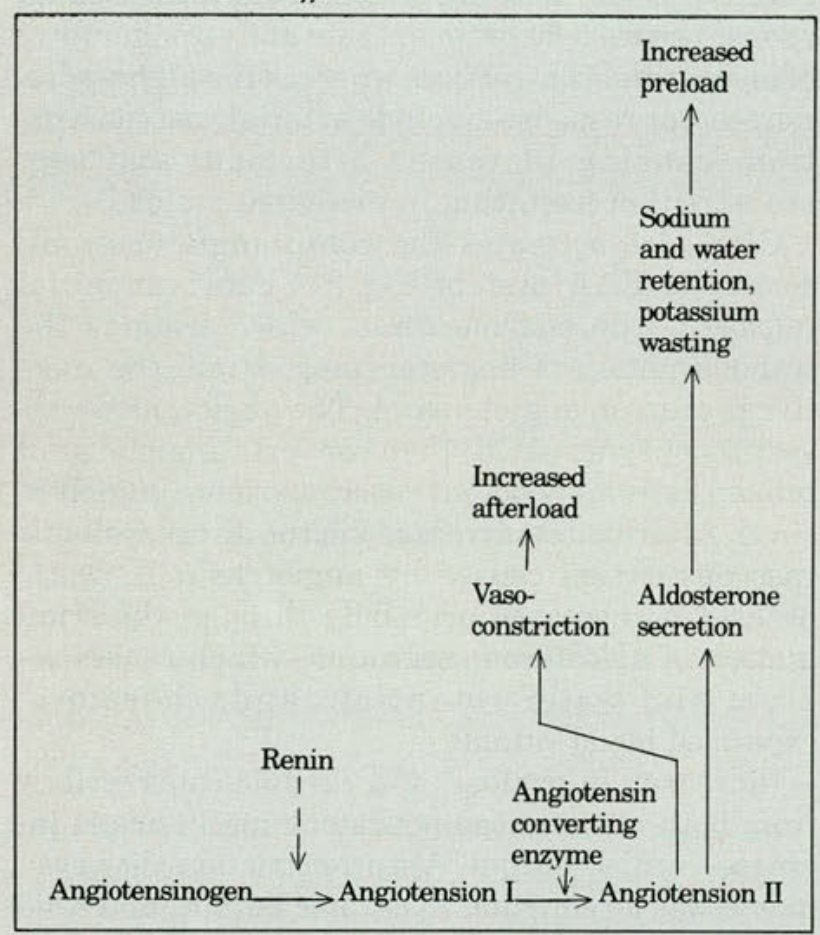

Fig. 2. RAA system in congestive heart failure. When left uninterrupted, the results are vasoconstriction, sodium and water retention, potassium wasting, and increased preload and afterload.

By interrupting the RAA system, captopril prevents the formation of angiotensin II, thereby decreasing vasoconstriction, systemic vascular resistance, and afterload. By decreasing (that is, normalizing) secretion of the sodium-retaining hormone aldosterone, captopril enhances sodium excretion, thus reducing volume expansion and preload. In addition to these two primary effects, captopril preferentially improves renal perfusion through vascular changes mediated by bradykinin and prostaglandins, to further improve sodium handling and diuresis. Via mechanisms that are not entirely clear, captopril therapy often corrects dilutional hyponatremia in patients with severe heart failure, which precludes the need for fluid restriction. It should be noted that despite improvements in renal plasma flow, captopril alone does not necessarily produce a diuresis, and it almost always should be used simultaneously with a diuretic. Captopril can markedly enhance the renal response to diuretics and significantly improve their diuretic action, thereby allowing for dosage reduction. Hemodynamically, the sum effects of captopril are reduced impedance (afterload) and increased cardiac output. The overall effects of treatment are improved total cardiac output, a favorable redistribution of regional blood flow (especially to the kidneys), decreases in total vascular resistance at rest and in heart rate, and, as sympathetic activity normalizes, enhanced vascular re-

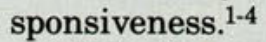

Clinically, captopril alleviates symptoms of dyspnea, fatigue, orthopnea, and edema and increases exercise capacity. ${ }^{5-8}$ The clinical course of 49 patients who received a regimen of digitalis, diuretics, and captopril in a randomized, double-blind multicenter trial $^{5}$ demonstrated significant al-

\begin{tabular}{|c|c|}
\hline Clinical effect & Patient benefits \\
\hline $\begin{array}{l}\text { Decreases serum levels of } \\
\text { angiotensin II }\end{array}$ & $\begin{array}{l}\text { Reduced peripheral re- } \\
\text { sistance, increased } \\
\text { cardiac output, and } \\
\text { improved symptoma- } \\
\text { tology and exercise } \\
\text { capacity }\end{array}$ \\
\hline Decreases aldosterone secretion & $\begin{array}{l}\text { Increased sodium excre- } \\
\text { tion, resulting in re- } \\
\text { duced fluid retention } \\
\text { and diuretic needs; } \\
\text { increased potassium } \\
\text { retention, resulting } \\
\text { in reduced or elimi- } \\
\text { nated need for po- } \\
\text { tassium supplements } \\
\text { or potassium-sparing } \\
\text { diuretics }\end{array}$ \\
\hline Improves renal perfusion & $\begin{array}{l}\text { Further enhanced so- } \\
\text { dium excretion, re- } \\
\text { sulting in further re- } \\
\text { duced diuretic needs }\end{array}$ \\
\hline Corrects dilutional hyponatremia & $\begin{array}{l}\text { Elimination of need for } \\
\text { fluid restriction }\end{array}$ \\
\hline Maintains long-term effectiveness & $\begin{array}{l}\text { Less visits for titration } \\
\text { of drug dose or re- } \\
\text { gimen modification; } \\
\text { fewer hospitalizations } \\
\text { for decompensated } \\
\text { CHF }\end{array}$ \\
\hline
\end{tabular}




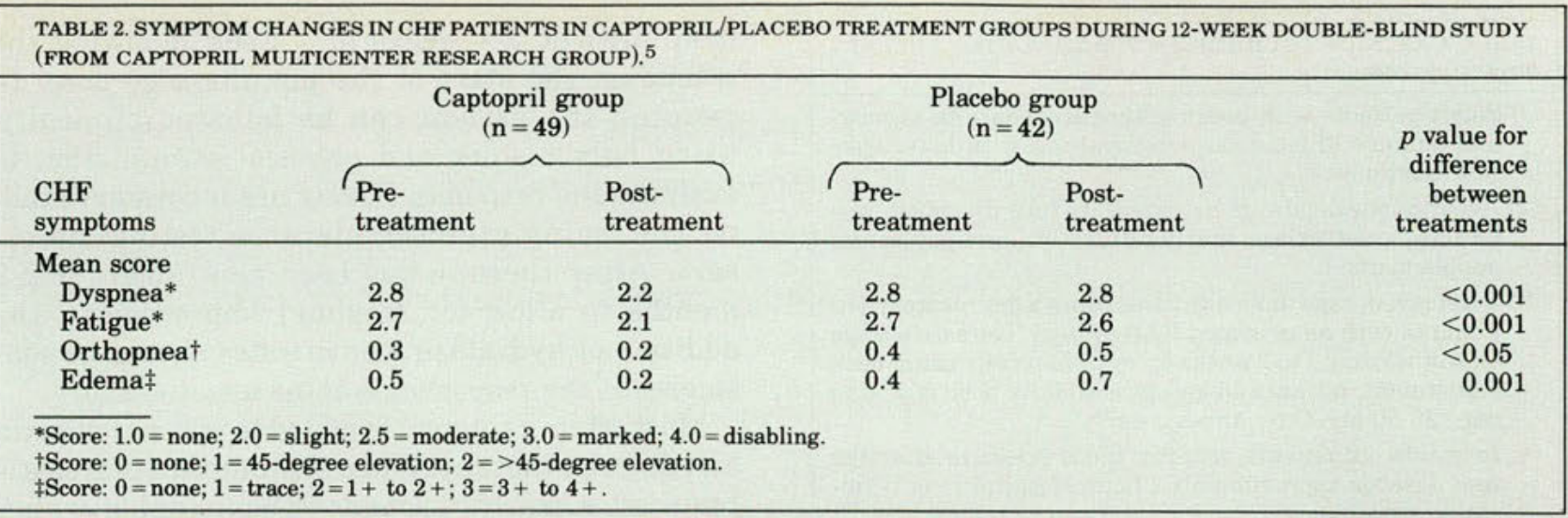

\begin{tabular}{|c|c|c|c|c|c|c|}
\hline \multirow{2}{*}{$\begin{array}{l}\text { NYHA } \\
\text { classifi- } \\
\text { cation } \\
\text { before } \\
\text { treatment }\end{array}$} & \multirow[b]{2}{*}{$\begin{array}{l}\text { No. of } \\
\text { patients }\end{array}$} & \multicolumn{5}{|c|}{$\begin{array}{l}\text { NYHA classification at final } \\
\text { evaluation }\end{array}$} \\
\hline & & I & IIS & IIM & III & IV \\
\hline I & 0 & 0 & 0 & 0 & 0 & 0 \\
\hline IIS & 0 & 0 & 0 & 0 & 0 & 0 \\
\hline IIM & 2 & 1 & 0 & 1 & 0 & 0 \\
\hline III & 45 & 3 & 15 & 15 & 11 & 1 \\
\hline IV & 55 & 1 & 6 & 17 & 23 & 8 \\
\hline
\end{tabular}

leviation of symptoms (Table 2) and increased exercise response (Fig. 3 ) as compared with 42 patients who received digitalis, diuretics, and placebo. The effects of captopril were accomplished through improved hemodynamics (sustained vasodilatation and increased cardiac output in association with reduced pulmonary and systemic venous congestion). Major side effects did not develop during captopril therapy in these patients. In a multicenter cooperative study, ${ }^{3} 124$ CHF patients who were resistant to digitalis and diuretic therapy were treated with captopril for varying periods of time, ranging from 1 week to 55 months. Those who had both pretreatment and post-treatment exercise stress testing exhibited a highly significant mean increase ( 34 percent) in exercise tolerance times. Importantly, a trend suggestive of increased survival time was observed. The effects of treatment on 102 patients who were classified according to New York Heart Association (NYHA) ratings subsequent to initial assessment are shown in Table 3.

\section{Clinical management}

Patients most likely to benefit from therapy with captopril, or any other vasodilator, are those who have left heart failure secondary to direct myocardial damage (infarction or myocarditis, for example) or left ventricular overload disorders

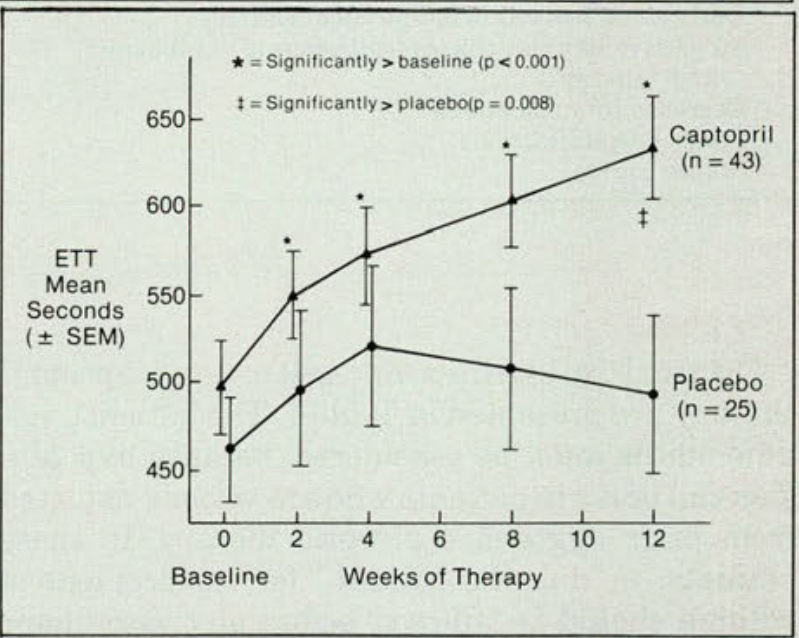

Fig. 3. Exercise response of patients with $\mathrm{CHF}$ that was refractory to digitalis and diuretics who received captopril or placebo. (Adapted from Captopril Multicenter Research Group 5 , with permission.)

(pressure- or volume-related). These patients have dilated, poorly contractile left ventricles, which usually respond to pharmacotherapy, in contrast to patients with heart failure caused by a restriction in ventricular filling (pericardial disease or mitral stenosis, for example). Patients with a restriction to ventricular filling show a poor response to medical treatment and, in many instances, do best with an appropriate surgical procedure, if this is possible. 
TABLE 4. CLINICAL GUIDELINES FOR INITIATING THERAPY WITH CAPTOPRIL.

(1) Select patients with heart failure secondary to myocardial damage (dilated cardiomyopathies with lower ejection fractions).

(2) Avoid hypovolemia. If necessary, reduce diuretics, vasodilators, potassium-sparing diuretics, and potassium supplements.

(3) Start low-dosage (6.25 mg. three times a day) captopril in patients with an activated RAA system. Increase dosage slowly, waiting 1 to 2 weeks for optimum response at each adjustment; advance to an optimal daily dose of 75-150 mg. (25-50 mg. three times a day).

(4) In stable outpatients, monitor blood pressure after the first dose for approximately 1 hour. Hospitalize only unstable patients.

(5) Check renal function and serum potassium level at 2 to 4 weeks or 1 week after maintenance dose of captopril is reached.

TABLE 5. ASSESSING RESPONSE TO CHRONIC THERAPY WITH CAPTOPRIL.

Objective measurements

Improvement in exercise ability

Improvement in renal function (weight loss, decrease in azotemia)

Correction of electrolyte abnormalities

Decrease in heart size

Quality of life considerations

Symptoms of dyspnea and fatigue

Daily activities requiring physical activity

Subjective impressions (overall sense of "well being" and "energy")

Decrease in medications

Fewer hospitalizations

Stable course
Clinical guidelines for initiating captopril therapy are presented in Table 4 . The patient's volume status must be considered, because hypotension can occur in patients who are volume-depleted from prior aggressive diuretic therapy. In these patients, a "diuretic holiday" for replacement of volume should be allowed before any vasodilator therapy is begun. For most patients, the usual starting dose should be $6.25 \mathrm{mg}$. three times a day. Effects on blood pressure after the first dose should be followed closely. In patients who are volume depleted or in those with a highly activated RAA system from other causes, an exaggerated initial hypotensive response may occur. (These patients often have a serum sodium level $<135 \mathrm{mEq}$./L.) The dose of captopril should be advanced to $12.5 \mathrm{mg}$. or $25 \mathrm{mg}$. three times a day in the ensuing 2 to 4 weeks in most patients. If necessary, the dose may be increased further; most patients show satisfactory clinical improvement at $25 \mathrm{mg}$. three times a day, or $50 \mathrm{mg}$. two/three times a day, and rarely are dosages greater than $150 \mathrm{mg}$./day required. Renal function and serum potassium values should be monitored at 2-4 weeks or 1 week following the extent on the start of the maintenance dose. In general, the patient can be followed clinically, using only history and physical examination to evaluate the response. Rarely are laboratory studies (including exercise-tolerance testing) necessary. After therapy has been continued for 2-3 months to allow for maximal improvement, the addition of hydralazine or nitrates should be considered if the response remains unsatisfactory.

Most often, captopril is added to optimal digoxin and diuretic therapy. When concomitant diuresis is required, a loop (furosemide or bumetanide) rather than a thiazide diuretic is preferred. For stable patients, captopril therapy may be initiated in the office without monitoring. The prompt absorption and action of captopril allow for the peak effects of treatment to appear early. Asking the patient to remain for approximately 1 hour after the initial dose of $6.25 \mathrm{mg}$. will enable the physician to evaluate hypotensive effects. Should symptomatic hypotension occur, laying the patient down will suffice (similar to treating the hypotensive effect from sublingual nitroglycerin). In unstable patients (those with severe angina or pre-existent hypotension), initiation of treatment may require hospitalization. If the patient experiences hypotensive or orthostatic symptoms, reduce the dosage of captopril or the diuretic. Discontinue captopril if symptoms continue or if azotemia develops. However, during two major clinical trials, ${ }^{3,5}$ only 3.6 percent of patients discontinued captopril therapy because of symptomatic hypotension.

If captopril is added to the regimen of a patient who is receiving other vasodilators, a significant decrease in blood pressure could result; consequently, caution is advised. Nitrates, alphablocking agents, or other smooth muscle dilators may be discontinued or reduced in dosage on the day when therapy with captopril is initiated, then subsequently titrated as indicated clinically.

In contrast to the short-acting captopril, the long-acting ACE inhibitor enalapril must be approached differently. Enalapril, which is in use in the U.S. for the treatment of hypertension, has been used investigationally in the U.S. in patients with $\mathrm{CHF}$ at dosages ranging from $2.5-20 \mathrm{mg}$. twice a day. A delayed and sustained hypotensive effect has been reported in CHF patients treated with enalapril. ${ }^{9}$ Occurring approximately $2-4$ hours post-treatment, this effect is due to the required metabolism by the liver of inactive enalapril to its active metabolite. The sustained hypotension has resulted in reduced renal perfusion and function, natriuresis, and significant increases in serum potassium levels. The time course of action of enal- 
april, with its later onset of action and appearance (peak effect 2-4 hours post-dosage), thus differs significantly from the earlier onset and peak effect $(<$ 2 hours) that may be seen following captopril administration.

Evaluation of CHF patients should always include assessment of renal function. Captopril is primarily excreted by the kidneys; it is therefore recommended that in renal-impaired patients daily dose be reduced, dosage increments for titration be smaller, and proceed slowly at 1 - to 2 -week intervals. After the desired therapeutic effect has been achieved, it may be possible in some patients to back-titrate slowly, so as to determine the lowest dosage at which efficacy can be demonstrated. However, $50-100 \mathrm{mg}$. per day appears to be the most common final daily dosage.

Enalaprilat, the active metabolite of enalapril, also is eliminated primarily through the kidney, and excretion rates are reduced in patients with impaired renal function. Furthermore, the conversion of enalapril to enalaprilat may be altered in patients with hepatic dysfunction and/or hepatic congestion. Few guidelines are presently available for the subgroup of patients with kidney and/or liver disorders, and it is prudent to be alert for necessary dosage reductions of enalapril in these patients.

Since ACE inhibitors decrease aldosterone secretion, potassium tends to be conserved. Therefore, patients should discontinue potassium-sparing agents (spironolactone, triamterene, amiloride) or potassium supplements when ACE inhibitor therapy is initiated. The need for such agents should be reassessed after therapy is maintained and if hypokalemia is confirmed. Often, a reduction in concurrent potassium supplementation is possible. Based on the individual needs of the patient, salt substitutes that contain potassium should also be advised or prohibited.

Diabetic patients with hypoaldosteronism and hyporeninemia do not appear to be at a significantly increased risk of developing hyperkalemia during captopril therapy. However, these patients and other diabetics with specific problems should be followed carefully. In a study ${ }^{10}$ that specifically evaluated patients with diabetic nephropathy and severe proteinuria, captopril therapy resulted in a decrease in urinary protein.

The minimum trial period of captopril should be 8 weeks, with progressive hemodynamic and functional improvements usually observed over the first 3 to 6 months. While other vasodilator drugs tend to lose their effectiveness through the appearance of tolerance during long-term administration in patients with severe heart failure,${ }^{11,12}$ the hemo- dynamic benefits achieved with captopril at 2-4 weeks after initiation generally are maintained and may become even more pronounced with time. ${ }^{2}$ Approximately 60 percent of patients treated with captopril can be expected to experience long-term symptomatic improvement. ${ }^{13}$ Because the initial hemodynamic response to captopril does not predict long-term efficacy, all patients who are candidates for therapy should be given a trial with the drug to determine its effectiveness. Some parameters for evaluating response are listed in Table 5.

\section{Comment}

Encouraging results from multicenter trials ${ }^{3,5}$ and other studies ${ }^{1,2,4,6-8,14-19}$ indicate that captopril, in producing both short- and long-term hemodynamic and clinical benefits, is effective in the treatment of CHF. These effects have been superior to the responses reported with other vasodilator drugs. Using the guidelines outlined in this paper, the primary care physician should find captopril to be a safe and effective therapy for patients with refractory CHF.

It has been well established that captopril can improve the quality of life in CHF patients. With the absence of long-term trials, it remains unclear whether patients live longer when treated with this drug, but there are suggestions of this possibility. ${ }^{20}$ The place of captopril as a first-line alternative to digitalis in CHF, as well as its potential influence on prognosis, are under present clinical investigation.

1. Cody, R.J., et al.: Captopril pharmacokinetics and the acute hemodynamic and hormonal response in patients with severe chronic con gestive heart failure. Am Heart J 104:1180-3, Nov 82

2. Ader, R., et al.: Immediate and sustained hemodynamic and clinical improvement in chronic heart failure by an oral angiotensin-converting enzyme inhibitor. Circulation 61:931-7, May 80

3. Captopril Multicenter Research Group I: A cooperative multicenter study of captopril in congestive heart failure. Hemodynamic effects and long-term response. Am Heart J 110:439-47, Aug 85

4. Kramer, B.L., Massie, B.M., and Topic, N.: Controlled trial of captopril in chronic heart failure. A rest and exercise hemodynamic study. Circulation 67:807-16, Apr 83

5. Captopril Multicenter Research Group: A placebo-controlled trial in refractory chronic congestive heart failure. J Am Coll Cardiol 2:755-63, Oct 83

6. Levine, T.B., Olivari, M.T., and Cohn, J.N.: Hemodynamic and regional blood flow response to captopril in congestive heart failure. Am J Med 76(513):38-42, 31 May 84

7. Sharpe, D.N., et al.: Low-dose captopril in chronic heart failure. Acute haemodynamic effects and long-term treatment. Lancet 2:1154-7, 29 Nov 80

8. Awan, N.A., et al.: Long-term hemodynamic and clinical efficacy of captopril therapy in ambulatory management of severe chronic congestive heart failure. Am Heart J 103:474-9, Apr 82

9. Packer, M., et al.: Comparison of captopril and enalapril in patients with severe chronic heart failure. N Engl J Med 315:847-53, 2 Oct 86 10. Taguma, Y., et al.: Effect of captopril on heavy proteinuria in azotemic diabetics. N Engl J Med 313:1617-20, 26 Dec 85 
11. Packer, M., et al.: Hemodynamic characterization of tolerance to longterm hydralazine therapy in severe chronic heart failure. N Engl J Med 306:57-62, 14 Jan 82

12. Bayliss, J., et al.: Clinical importance of the renin-angiotensin system in chronic heart failure. Double blind comparison of captopril and prazosin. Br Med J 290:1861-5, 22 Jun 85

13. Packer, M.: Vasodilator and inotropic therapy for severe chronic heart failure. Passion and skepticism. J Am Coll Cardiol 2:841-52, Nov 83

14. Dzau, V.J., et al.: Sustained effectiveness of converting-enzyme inhibition in patients with severe congestive heart failure. N Engl J Med 302:1373-9, 19 Jun 80

15. Packer, M., Medina, N., and Yushak, M.: Contrasting hemodynamic responses in severe heart failure. A comparison of captopril and other vasodilator drugs. Am Heart J 104:1215-23, Nov 82

16. Cleland, J.G.F., et al.: Total body and serum electrolyte composition in heart failure. The effects of captopril. Eur Heart J 6:681-8, 1985

17. Rouleau, J.L., et al.: Alterations in left ventricular function and coronary hemodynamics with captopril, hydralazine and prazosin in chronic ischemic heart failure. A comparative study. Circulation 65:671-8, Apr 82

18. LeJemtel, T.H., et al.: Hemodynamic effects of captopril in patients with severe chronic heart failure. Am J Cardiol 49:1484-8, 21 Apr 82

19. Romankiewicz; J.A., et al.: Captopril. An updated review of its pharmacological properties and therapeutic efficacy in congestive heart failure. Drugs 25:6-40 Jan 83

20. Furberg, C.D., and Yusuf, S.: Effect of vasodilators on survival in chronic congestive heart failure (editorial). Am J Cardiol 55:1110-3, 1 Apr 85

Dr. DiBianco is director of cardiovascular research, Washington Adventist Hospital, and associate clinical professor of medicine, Georgetown University, Washington, D.C.

Dr. DiBianco, Washington Adventist Hospital, 7600 Carroll Avenue, Takoma Park, Maryland, 20912. 